\title{
La cobertura del cultivo como indicador de la productividad de maíz en un lote con siembra variable
}

\author{
de la Casa, A.; G. Ovando, L. Bressanini, J. Martínez, C. Miranda y G. Díaz
}

\begin{abstract}
RESUMEN
La variabilidad del rendimiento de un lote se puede monitorear por diversos medios remotos. El objetivo del trabajo fue determinar la cobertura del cultivo (CC) de maíz (Zea mays L.) a partir de datos de NDVI, para evaluar las diferencias productivas en un lote de 45 ha bajo riego ubicado al sur de la ciudad de Córdoba, Argentina, durante el ciclo 2013-2014. En el lote se establecieron seis sectores de muestreo donde periódicamente fueron adquiridas fotografías con una cámara digital que produce datos de NDVI. A los efectos de determinar CC, las imágenes de NDVI se clasificaron aplicando distintos umbrales para discriminar entre píxeles con vegetación y suelo. Cualquiera de los umbrales utilizados permitió establecer diferencias de CC entre sectores que se incrementaron durante la etapa reproductiva. El contraste entre los valores de NDVI locales y los de AQUA-MODIS mostró relaciones lineales muy consistentes, que permite distinguir un potencial productivo diferente entre los sectores norte y sur del lote. El contenido de agua del suelo acusó diferencias significativas $(P<0,01)$ entre los sectores durante las etapas R1 y R4 que, concurrente con la disminución de CC y la menor densidad de siembra, explicaría la merma productiva al norte del lote de maíz.
\end{abstract}

Palabras clave: agricultura de precisión, NDVI, biomasa, agua del suelo.

de la Casa, A.; G. Ovando, L. Bressanini, J. Martínez, C. Miranda and G. Díaz, 2017. Crop cover as indicator of corn productivity in a field with variable seeding. Agriscientia 34: 1-14

\section{SUMMARY}

Yield variability within field can be monitored by various remote sensing technologies. The objective of this study was to determine the crop cover (CC) of corn (Zea mays L.) from NDVI data obtained from digital photographs, looking for productivity differences between sectors in an irrigated field of 45 ha located south of Córdoba city, Argentina, during 2013-2014. In this field six sampling sectors were set and digital photos were taken periodically in them with a modified camera to produce NDVI data. The CC estimation was conducted using different thresholds to NDVI values to discriminate between pixels with vegetation and soil. A different behavior of CC was observed in the six sectors whatever the threshold that was used, and these differences were 
more pronounced during the reproductive stage. The contrast of local NDVI values with those of AQUA-MODIS showed very consistent linear relationships and allowed to distinguish different productive sectors between the north and the south of the field. Soil water content accused significant differences ( $P$ $<0.01$ ) between the north and south sectors during R1 and R4 stages, which concurrent with the decrease in CC and the lower density of planting, could explain the lower production in the northern sectors of the field.

Key words: precision agriculture, NDVI, biomass, soil water.

A. de la Casa, G. Ovando, G. Díaz, L. Bressanini y J. Martínez: Agrometeorología, Agrotecnia y Física de la Facultad de Ciencias Agropecuarias (UNC), Córdoba CP: 5000, Argentina. C. Miranda: Establecimiento Las Delicias. Correpondencia a: delacasa@agro.unc.edu.ar

\section{INTRODUCCIÓN}

La variabilidad ambiental y productiva dentro de un lote es la condición necesaria que justifica la agricultura de precisión (AP) (Mulla and Schepers, 1997). Las diferencias de productividad del lote, que se expresan de manera definitiva en el mapa de rendimiento, pueden ocurrir tanto por la capacidad productiva propia de cada sector, por contingencias ambientales/productivas de cada ciclo, como por decisiones de manejo. A los efectos de comprender las causas de la variabilidad se requiere monitorear el crecimiento y desarrollo del cultivo durante el ciclo en distintos sectores/ambientes, tarea que se puede realizar a partir de mediciones de reflectancia espectral por diversos medios remotos (Hatfield et al., 2008).

La cobertura verde o fotosintetizante del cultivo (CC) es un parámetro biofísico apropiado para caracterizar su crecimiento y desarrollo ya que permite estimar tanto la tasa de transpiración como la producción de biomasa de la vegetación. En el primer caso, la variación de CC a lo largo del ciclo permite discriminar en el proceso de evapotranspiración, la evaporación de agua del suelo respecto a la que transpira un cultivo y que, por esa razón, tiene mayor carácter fisiológico (Kool et al., 2014). En el segundo, constituye un factor que se vincula directamente con la fracción de radiación solar fotosintéticamente activa interceptada (fRFAl) por el dosel (de la Casa et al., 2007; 2011). La relación entre la materia seca producida y la radiación interceptada por el cultivo en un periodo determinado se denomina eficiencia en el uso de la radiación (EUR, $\mathrm{g} \mathrm{MJ}^{-1}$ ) (Lindquist et al., 2005). Por su parte, Steven et al. (1986) y Purcel (2000) utilizan la CC para representar de manera directa el valor de fRFAl. El monitoreo continuo de
CC durante el ciclo del cultivo puede proveer información para estimar la producción de biomasa en maíz sembrado a tasa variable, como así también permitiría explicar las causas determinantes de la variación espacial de los rendimientos en el lote.

En razón de la mayor versatilidad y facilidad para su obtención a campo, el modelo de cultivo AquaCrop estructura las funciones de crecimiento y desarrollo de acuerdo a la variación de CC a lo largo del ciclo (Raes et al., 2009). En otro sentido, los procedimientos que ensamblan los modelos de cultivo con la teledetección, por lo general emplean tanto el índice de área foliar como la CC para realizar el acople de ambas tecnologías (de la Casa, 2011). Así, a partir de la información satelital que tiene un alcance espacial más generalizado, es factible corregir la simulación del modelo en tiempo y forma para conseguir mayor precisión en las estimaciones de productividad (Maas, 1993; Inoue, 2003; Doraiswamy et al., 2004).

Algunos índices de vegetación utilizan sólo las bandas espectrales rojo $(r)$, verde $(v)$ y azul (a) del visible, adquiridas tanto de instrumentos montados en satélites como directamente sobre el cultivo, para estimar la fracción de suelo cubierta por el cultivo (CC). Woebbecke et al. (1995) mostraron que el índice de vegetación de exceso de verde (ExG) permite identificar malezas bajo distintos tipo de suelo, cobertura de residuos y condiciones de iluminación. Con información espectral de campo, Gitelson (2002) desarrolló algoritmos como el índice del visible resistente atmosféricamente (VARI, por sus siglas en inglés) que utiliza sólo datos del visible para estimar la fracción de cobertura.

Por su parte, a partir de los datos espectrales del rojo $(r)$ e infrarrojo cercano (nir) de imágenes Landsat 5 TM, la misma información que utiliza el índice de 
vegetación de la diferencia normalizada (NDVI), Maas y Rajan (2008) presentan un procedimiento para estimar CC basado en un modelo de mezclado lineal. Utilizando datos de NDVI del sensor Moderate Resolution Imaging Spectroradiometer (MODIS) de resolución media, de la Casa et al. (2013a) determinaron por correlación y regresión un modelo para estimar CC en cultivos de maíz a escala de lote. Las mediciones de CC en ese caso se obtuvieron a campo a partir de imágenes digitales convencionales adquiridas sobre el cultivo que fueron sometidas a un procesamiento supervisado de clasificación de máxima verosimilitud. De la Casa et al. (2013b) también emplearon el cociente entre las bandas verde $(v)$ y roja $(r)$ de fotografías digitales convencionales para estimar CC en papa, reportando alguna limitación del procedimiento al presentarse la senescencia.

Hunt et al. (2010), por su parte, utilizan una cámara fotográfica convencional (Fuji FinePix S3 Pro UVIR) que fue modificada para obtener imágenes digitales integrando los canales nir, $v$ y a. A partir de esta información radiométrica elaboran un índice de vegetación y, con la cámara montada en un vehículo aéreo no tripulado (UAV, por sus siglas en inglés), realizan un monitoreo detallado del estado de los cultivos a escala de lote. De igual forma, también se considera factible estimar CC directamente sobre el terreno utilizando una cámara fotográfica comercial con su sistema óptico modificado para obtener datos de NDVI. Los resultados, restringidos en este caso a la escala local, se obtienen estableciendo en el algoritmo de clasificación un umbral de NDVI destinado a identificar cada píxel de la imagen como vegetación o suelo (de la Casa et al., 2013b).

La AP integra un conjunto de tecnologías destinadas a optimizar la producción a partir de un manejo localizado en cada sector del lote (Pierce y Nowak, 1999). Moran et al. (1997) consideran distintas alternativas de uso de los sensores remotos para proveer información destinada a mejorar la implementación de las aplicaciones del manejo de precisión. Entre otras utilidades, la teledetección se ha empleado de manera operativa para realizar pronósticos de cosecha (Teal et al., 2006). En su forma más simple, el rendimiento final de un cultivo se correlaciona con una observación individual del NDVI o sus valores integrados durante periodos específicos del ciclo de cultivo. Esta información radiométrica se ha aplicado, además, para identificar las etapas fenológicas del cultivo de maíz y sus cambios durante el ciclo (Viña at al., 2004; Martin et al., 2007), como así también para evaluar las recomendaciones de fertilización nitrogenada. Dichas recomendaciones basadas en valores de NDVI obtenidos en la eta- pa vegetativa entre V8 y V9 resultaron más precisas que cuando se utilizó la información de productividad (Clay et al., 2006).

Una práctica de manejo específica de la AP consiste en modificar la densidad de siembra de acuerdo al potencial productivo de cada lugar. La densidad de siembra está ligada directamente con la población de plantas y, en consecuencia, a la mayor o menor intensificación en el uso de los recursos disponibles (Woli et al., 2014). En este sentido, el monitoreo de CC a lo largo del ciclo, obtenida tanto desde plataformas satelitales (Maas, 1998; 2000) como con instrumentos a campo (Gitelson, 2004), puede ser útil a los efectos de expresar el potencial productivo y, de esta forma, identificar aquellos sectores del lote que presentan limitaciones.

En este trabajo se emplean valores de CC estimados a partir de registros de NDVI de una cámara digital para representar el potencial productivo de distintos sectores de un lote de maíz que, con el propósito de aprovechar las ventajas del manejo localizado y a partir de información previa, fue sembrado a tasa variable. Por otra parte, se evalúa la influencia del umbral empleado para discriminar entre material vegetal verde y el suelo en el procedimiento de clasificación de imágenes a fin de estimar CC. Asimismo, estos datos locales de NDVI obtenidos sobre el cultivo con mayor detalle espacial son contrastados con información similar del sensor MODIS que, si bien tiene una resolución espacial moderada (un píxel de $250 \mathrm{~m}$ ) que sólo puede ofrecer una visión panorámica del lote, permite disponer de una referencia independiente sobre la calidad de la información de campo. Finalmente, el comportamiento productivo monitoreado se evalúa y las diferencias entre sectores son interpretadas a partir del conjunto de evidencia recogida.

\section{MATERIALES Y MÉTODOS}

\section{Detalles del ensayo}

El estudio se llevó a cabo en un cultivo de maíz (Zea mays L.) de la variedad MK 960 - TD, TG de SINGENTA®, a partir de datos relevados entre el 4/10/2013 (siembra) y el 11/02/2014 (R6). La cosecha se demoró hasta el 24/04/2014 cuando la humedad del grano resultó apropiada. Las mediciones se realizaron en un lote de 45 ha del establecimiento Las Delicias ubicado al sur de la ciudad de Córdoba ( $31^{\circ} 33^{\prime} \mathrm{S}, 64^{\circ} 05^{\prime} \mathrm{O}, 400 \mathrm{~m}$ s.n.m), en cuya proximidad se encuentra instalada una estación meteorológica automática que proveyó los datos para evaluar las condiciones atmosféricas durante el ensayo. El suelo del predio es un Haplustol éntico, serie Manfredi, cuyo perfil 
presenta tres horizontes de textura franco-limosa (Rollán \& Bachmeier, 2014). A partir de un mapa de rendimiento de un cultivo de soja de secano de la campaña 2011/12, se delimitó una franja de mayor productividad potencial hacia el sur (S), donde se sembró a razón de 10 semillas $\mathrm{m}^{-2}$, y otra hacia el norte $(\mathrm{N})$ del lote, utilizando 8 semillas $\mathrm{m}^{-2}$. En cada una de las franjas se establecieron tres áreas de muestreo: una próxima a la cabecera de riego (Oeste), otra en el centro (C) y la restante el final o cola del surco (Este). De esta forma, los sectores de muestreo se establecieron territorialmente de acuerdo a su posición geográfica (SO, SC, SE, NO, NC y NE), como muestra la Figura 1.

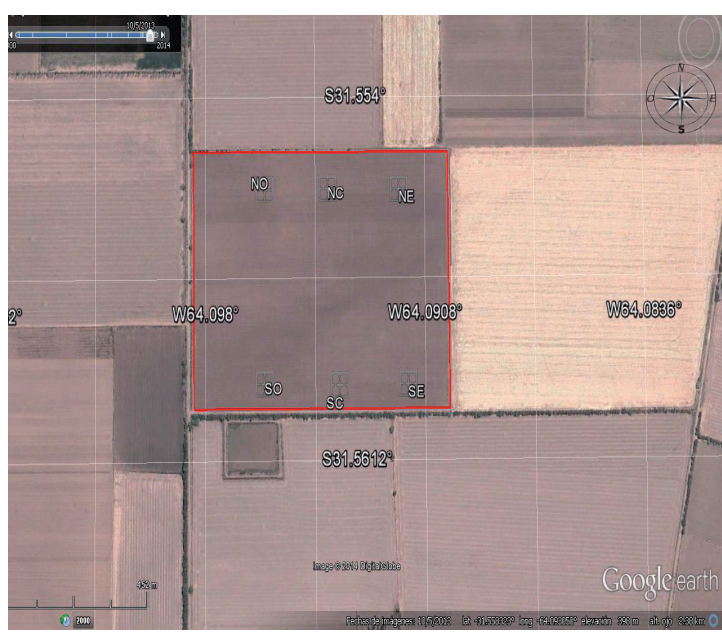

Figura 1. Posición geográfica del lote de maíz (perímetro en rojo) al sur de la ciudad de Córdoba (Argentina) y ubicación de los seis sectores de muestreo durante el ciclo 2013-2014 (Imagen de Google Earth de 5/10/2013).

El cultivo fue fertilizado a la siembra con 150 $\mathrm{kg} \mathrm{ha}^{-1}$ de urea, $150 \mathrm{~kg} \mathrm{ha}^{-1}$ de sulfato de amonio, y posteriormente con Zn por vía foliar. Además, recibió cinco riegos por surco de $80 \mathrm{~mm}$ de agua cada uno, tres antes de floración y dos después (Figura 2). En cada una de las ocho fechas de muestreo, para cada sector se procedió a medir la cantidad de plantas en 5 metros lineales de dos filas contiguas elegidas al azar a fin de obtener la población de plantas, y se extrajo la parte aérea de dos de ellas para determinar la materia seca total de acuerdo a la población de cada sector. El contenido de agua del suelo se registró utilizando el método gravimétrico, a partir de un sondeo del perfil a cinco profundidades $(0,1 ; 0,3 ; 0,7 ; 0,9$ y 1,1 $\mathrm{m})$. Estas mediciones se integraron a los efectos de obtener el contenido de agua del perfil hasta 1,2 $\mathrm{m}$, considerando un valor de densidad aparente de $1,25 \mathrm{Mg} \mathrm{m}^{-3}$ (Rollán \& Bachmeier, 2014). El contenido de humedad se expresó en porcentaje respecto del valor a capacidad de campo de 0,32 $\mathrm{m}^{3} \mathrm{~m}^{-3}$ (Rollán \& Bachmeier, 2014).

Los datos provistos por el monitor de cosecha al final del ciclo fueron representados en un mapa de rendimiento y se analizaron con respecto a la información obtenida en los sitios de muestreo. Por medio de un ANAVA se estableció la diferencia entre sectores.

\section{Datos NDVI de la cámara digital modificada}

La CC se determinó a partir de fotografías adquiridas con una cámara Canon Powershot SD780 IS, modificada para producir imágenes de NDVI (de la Casa et al., 2013b) en resolución VGA (640x480 píxeles). Hunt et al. (2010) emplean un dispositivo similar para realizar el monitoreo de los cultivos a escala de lote montando la cámara en un avión no tripulado. Las fotografías se tomaron a $2 \mathrm{~m}$ por encima del cultivo, altura que se mantuvo constante a lo largo del ciclo por medio de un dispositivo extensible. De acuerdo a esto, la superficie analizada a la altura del cultivo fue aproximadamente de 5,45 $\mathrm{m}^{2}$. La determinación tradicional de NDVI, procesando datos del infrarrojo cercano (nir) y el rojo ( $r$ ) en el visible del espectro electromagnético, usa la fórmula:

$$
N D V I=\frac{(\text { nir }-r)}{(\text { nir }+r)}
$$

Las cámaras digitales obtienen por separado las distintas bandas del espectro, de modo que una vez aislada la banda nir a través de un filtro específico y, localizada esta señal en una de las bandas de color, es posible obtener una medición del tipo NDVI. El modelo de cámara que se utilizó para este propósito registra el canal azul (a) y el canal verde $(v)$ de la luz visible, mientras que el rojo corresponde al nir. De esta forma, la fórmula del NDVI puede ser reescrita como:

$$
B N D V I=\frac{(\text { nir }-a)}{(\text { nir }+a)}
$$

Mayores detalles técnicos de esta cámara modificada pueden consultarse en von Bueren et al. (2015). Respecto al empleo de distintas bandas para elaborar el índice de vegetación, Wang et al. (2007) compararon valores de NDVI calculados a partir de un radiómetro en un lote con arroz, con valores de NDVI que fueron reemplazados por la banda roja por la azul (BNDVI) y por la verde (GNDVI), y encontraron valores de correlación de 0,981 entre BNDVI y GNDVI y de 0,987 entre 


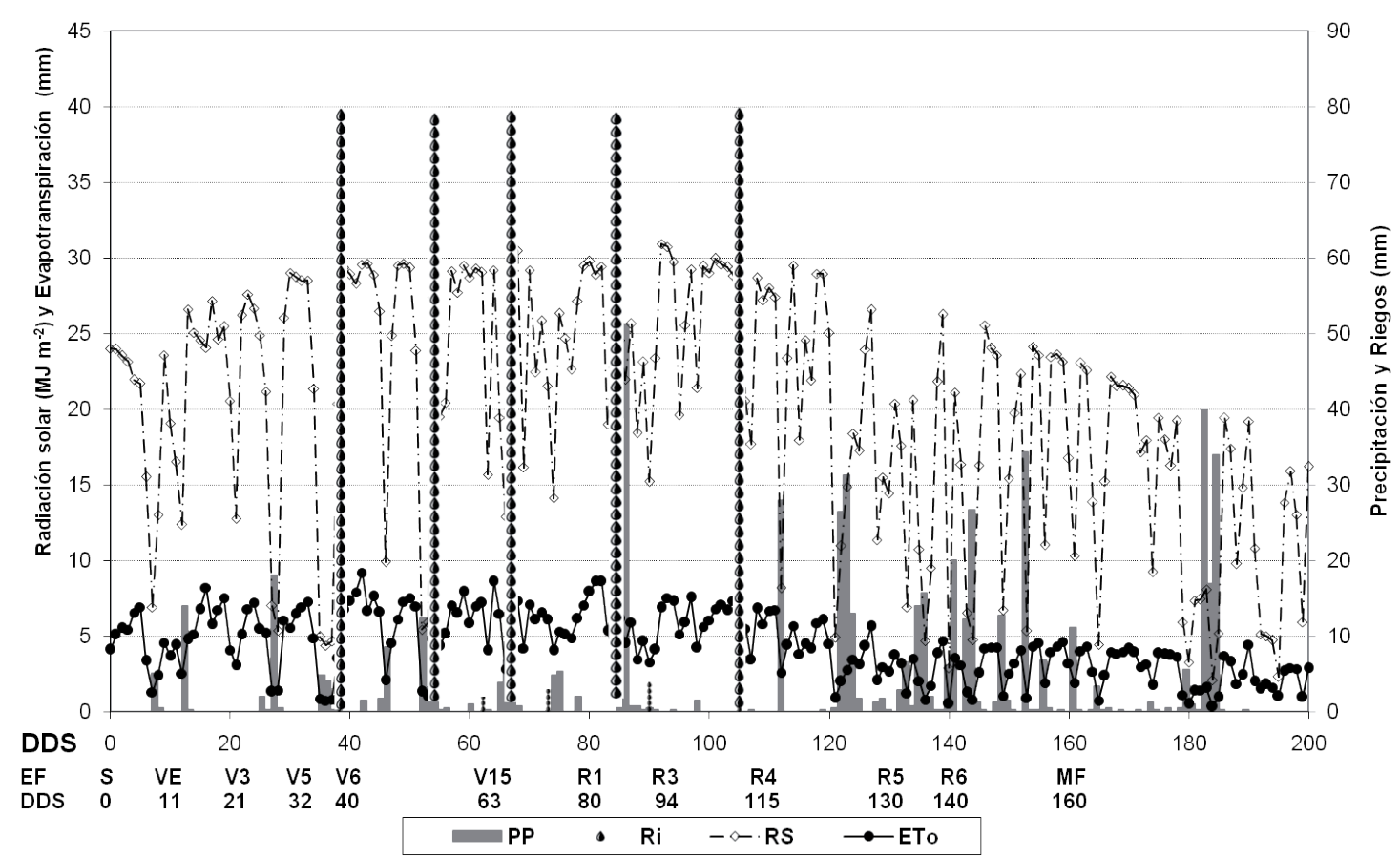

Figura 2.Variación de la precipitación (PP), riegos suministrados (Ri), radiación solar (RS) y evapotranspiración de referencia (ETo) en relación a los días después de la siembra (DDS) de maíz. Se indica abajo la secuencia de etapas fenológicas (EF) observadas de acuerdo a la escala de Ritchie et al. (1992).

BNDVI y NDVI.

La obtención de CC requiere diferenciar en la imagen dos clases: suelo o vegetación verde y, a partir de esta imagen clasificada, calcular la relación entre la cantidad de pixeles con vegetación con respecto al total, expresada como fracción (01) o porcentaje (0-100\%). Los umbrales utilizados para clasificar las imágenes fueron 0,$0 ; 0,05 ; 0,1$ y 0,15 , y se consideró como suelo a los píxeles con valores de BNDVI inferiores al umbral. En tal sentido Li et al. (2010) encontraron una relación lineal significativa entre la CC estimada a partir de una cámara digital convencional con un umbral de 0,0 y valores de NDVI de GreenSeeker ${ }^{\circledR}$, que considera las bandas del rojo e infrarrojo cercano. Tanto para el cálculo de BNDVI como para la clasificación y determinación de CC, en este trabajo se desarrolló un programa en lenguaje IDL v7.0.8.

\section{Datos NDVI de AQUA-MODIS}

La información radiométrica de origen satelital utilizada corresponde al producto MYD13Q1 del sistema satélite /sensor AQUA-MODIS y fue obtenida directamente del portal de EROS Data Center (EOS, 2014). Se emplearon estas imágenes en lugar de utilizar las del satélite TERRA, debido a la menor dispersión que presentan sus ópticas y mejor respuesta del espejo de barrido respecto del ángulo de escaneo (Xiong \& Barnes, 2003). De este producto sólo se procesaron los datos de NDVI, que tienen una resolución espacial de $250 \mathrm{~m}$ y corresponden a una composición de 16 días. Este índice de vegetación utiliza valores de reflectancia superficial corregidos por dispersión molecular, absorción del ozono y aerosoles, y están ajustados al nadir mediante el empleo de un modelo BRDF (Huete et al., 2002). La resolución espacial de $250 \mathrm{~m}$ del sensor MODIS determina un píxel que integra aproximadamente 6,25 ha, de modo que esta información presenta estrictamente un alcance general para el lote de 45 ha, razón por la cual se consideraron sólo 4 píxeles centrales.

La alternativa de recurrir a registros de Landsat para estudios a escala de lote que ofrece mayor precisión (Sibley et al., 2013), no se pudo implementar en esta ocasión porque la nubosidad persistente hacia el final del ciclo impidió disponer de información útil. 


\section{RESULTADOS Y DISCUSIÓN}

El cultivo de maíz monitoreado durante el ciclo 2013-2014 experimentó las condiciones agrometeorológicas que, en los valores diarios de precipitación, radiación solar y evapotranspiración de referencia, muestra la Figura 2. La lluvia fue escasa en los primeros estadíos del cultivo, acumulando hasta la fecha de floración (80 días después de siembra) $106 \mathrm{~mm}$, de modo que la falta de agua se corrigió mediante la aplicación de riego. A partir del día 80 los eventos de lluvia aumentaron su frecuencia hasta totalizar al final del ciclo (200 DDS) 550 mm, lo que produjo una disminución marcada tanto de la radiación solar como de la demanda atmosférica de agua durante la etapa de llenado de grano.

A partir del recuento de plantas efectuado durante el ciclo, los sectores de muestreo presentaron la población que muestra la Figura 3. El análisis de varianza indica que este factor presentó diferencias significativas $(P<0,001)$ entre sectores, lo que se justifica en tanto la siembra se realizó de manera diferenciada de acuerdo a la aptitud que se observó en el mapa de rendimiento de un cultivo de soja en 2011. Mientras la densidad de plantación media en la franja norte oscila entre 7,8 y $8,3 \mathrm{pl} \mathrm{m}^{-2}$, los sectores del sur presentan valores medios iguales o superiores a $8,9 \mathrm{pl} \mathrm{m}^{-2}$; con esto se constató que la diferencia de la población de plantas a la siembra se mantuvo a lo largo del ciclo de cultivo. La población de plantas en los sectores del sur estaría comprendida dentro del rango que produce el rendimiento de grano máximo en la región. En tal sentido, Woli et al. (2014) alcanzaron el máximo rendimiento de $13,4 \mathrm{Mg} \mathrm{ha}^{-1}$ con una tasa de 96000 semillas ha-1, valor óptimo que estaría sujeto a las condiciones meteorológicas, edáficas y de manejo de cada lugar.

La Figura 4 presenta la variación de la CC en cada uno de los seis sectores del lote de maíz monitoreado durante el ciclo 2013-2014, valores que se obtuvieron considerando distintos umbrales para clasificar las imágenes originales de BNDVI. Independientemente del umbral establecido, la CC es siempre más reducida a partir de 50 días de la siembra (DDS) en los tres sectores de muestreo ubicados sobre la franja norte del lote, en correspondencia inicialmente a la menor densidad de siembra que se había dispuesto.

Teniendo en cuenta que el cultivo de maíz presenta un crecimiento determinado, el ciclo completo se puede dividir en dos subperiodos (Ritchie et al., 1992): el vegetativo desde siembra hasta la aparición de la panoja masculina, que en este caso se presentó aproximadamente a los 80 DDS, y el reproductivo desde aparición de estigmas hasta la madurez fisiológica (Viña et al.,

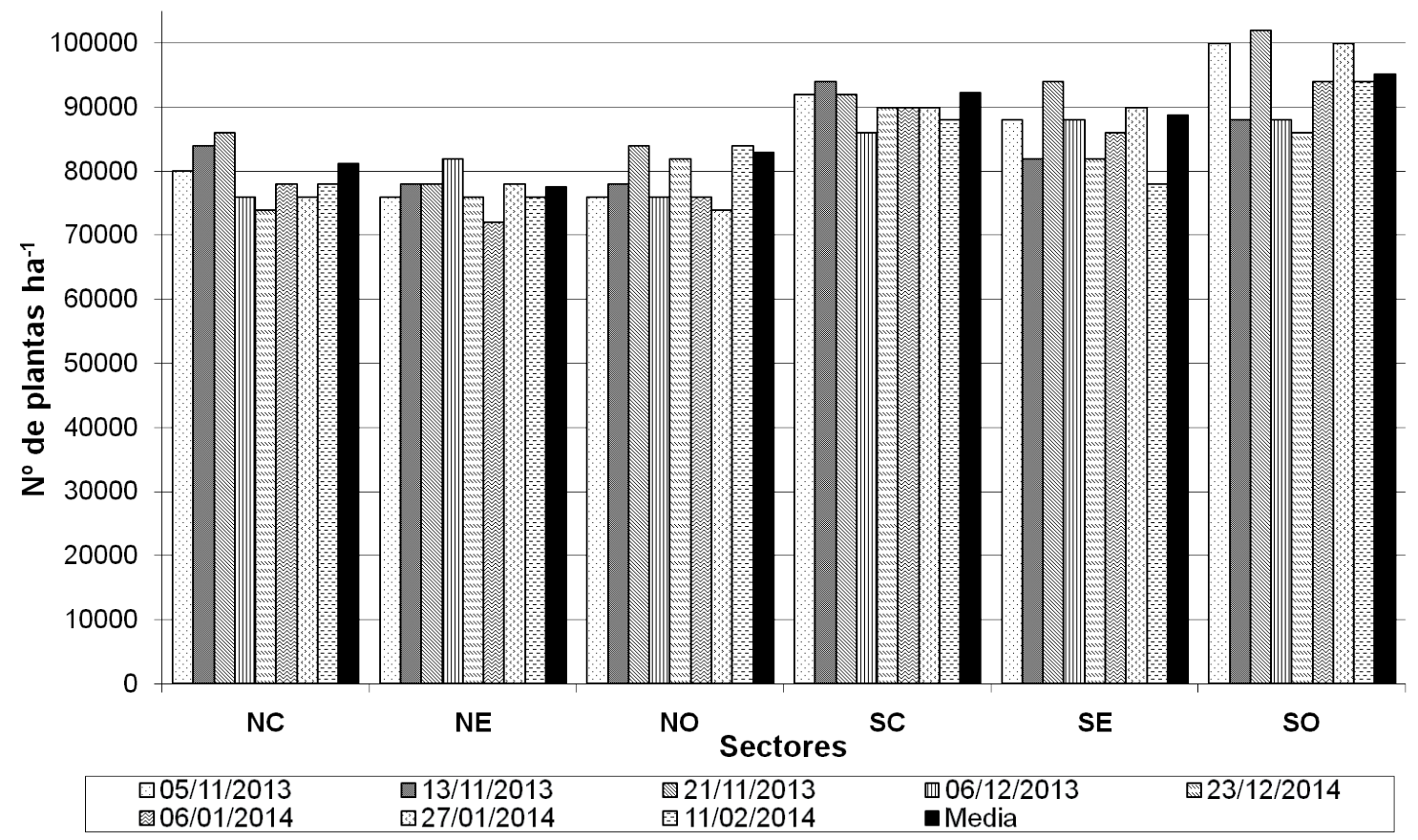

Figura 3. Población de plantas para cada sector en las distintas fechas de muestreo. 

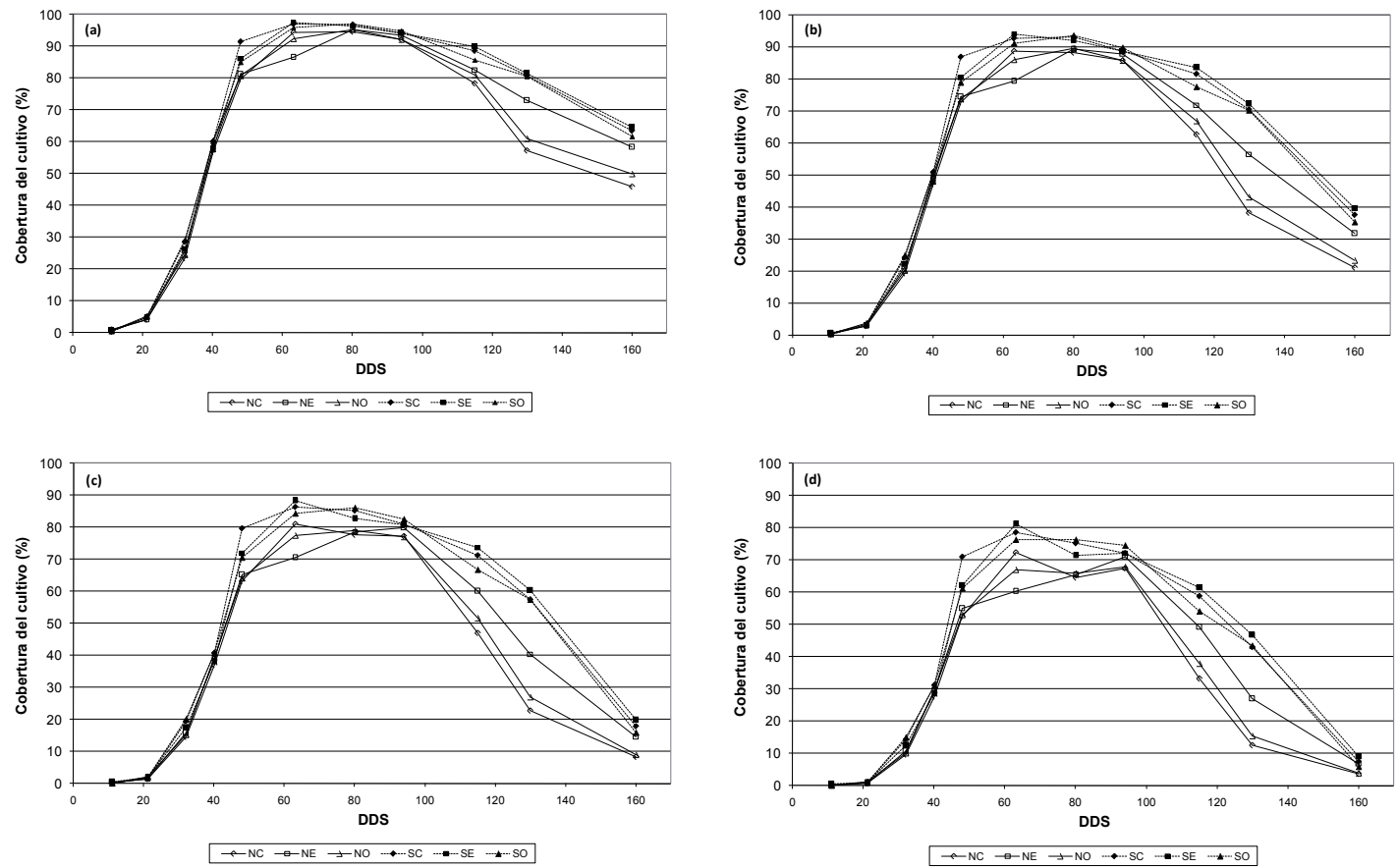

Figura 4. Variación de la cobertura del cultivo estimada con los umbrales 0,0 (a); 0,05 (b); 0,1 (c) y 0,15 (d) respecto a los días después de la siembra (DDS) en los distintos sectores del lote de maíz durante el ciclo 2013-2014 en Córdoba.

2004). Mientras la diferencia de los valores de CC en el subperiodo de crecimiento vegetativo del maíz resulta más atenuada entre los distintos sectores, en el subperiodo reproductivo las diferencias se incrementan notablemente. Cualquiera sea el umbral de clasificación considerado, la mayor caída que registra CC en los tres sectores de la franja hacia el norte del lote pone de manifiesto una eventual merma productiva durante esa etapa, más allá de las diferencias en la densidad de siembra.

El trazado de las curvas de CC presenta diferencias sistemáticas, con valores más reducidos a medida que se incrementa el umbral de clasificación. Con relación a la selección del umbral más adecuado para estimar CC, y de acuerdo a la inspección visual realizada durante el ciclo, es razonable descartar los valores que se obtuvieron hacia el final con los umbrales de 0,0 y 0,05 , ya que el cultivo en madurez fisiológica no presentaba tejido de apariencia verde, resultando la CC seriamente sobreestimada en ese caso. Hasta tanto no se pueda convalidar fehacientemente este comportamiento, a los efectos de utilizar los valores de CC y proyectarlos para estimar la producción de biomasa y rendimiento, se considera apropiado combinar los resultados de modo que en la etapa vegetativa el umbral sea 0,0 o 0,05 , ya que el cultivo alcanza efectivamente valores de CC próximos a 100\%. Por su parte, en la etapa reproductiva se considera más consistente clasificar las imágenes a partir de los umbrales más elevados para expresar la ausencia de material fotosintéticamente activo. No obstante, cualquiera sea el umbral de clasificación que se utilice a los efectos del cómputo los resultados avalan en términos relativos el empleo de la CC obtenida a campo para expresar diferencias dentro del lote, producidas ya sea por la densidad de siembra o como consecuencia de un diferente potencial productivo.

\section{Consistencia entre los datos de NDVI obteni- dos a campo y de origen satelital}

A los efectos de analizar el alcance de los datos obtenidos a campo con la cámara digital modificada, los registros BNDVI se contrastaron con datos de NDVI de origen satelital en su carácter de información independiente. La Figura 5 (a) muestra la variación temporal de los registros de NDVI durante el ciclo de maíz obtenidos alternativamente por el sistema de teledetección AQUA-MODIS y por la cámara digital directamente 

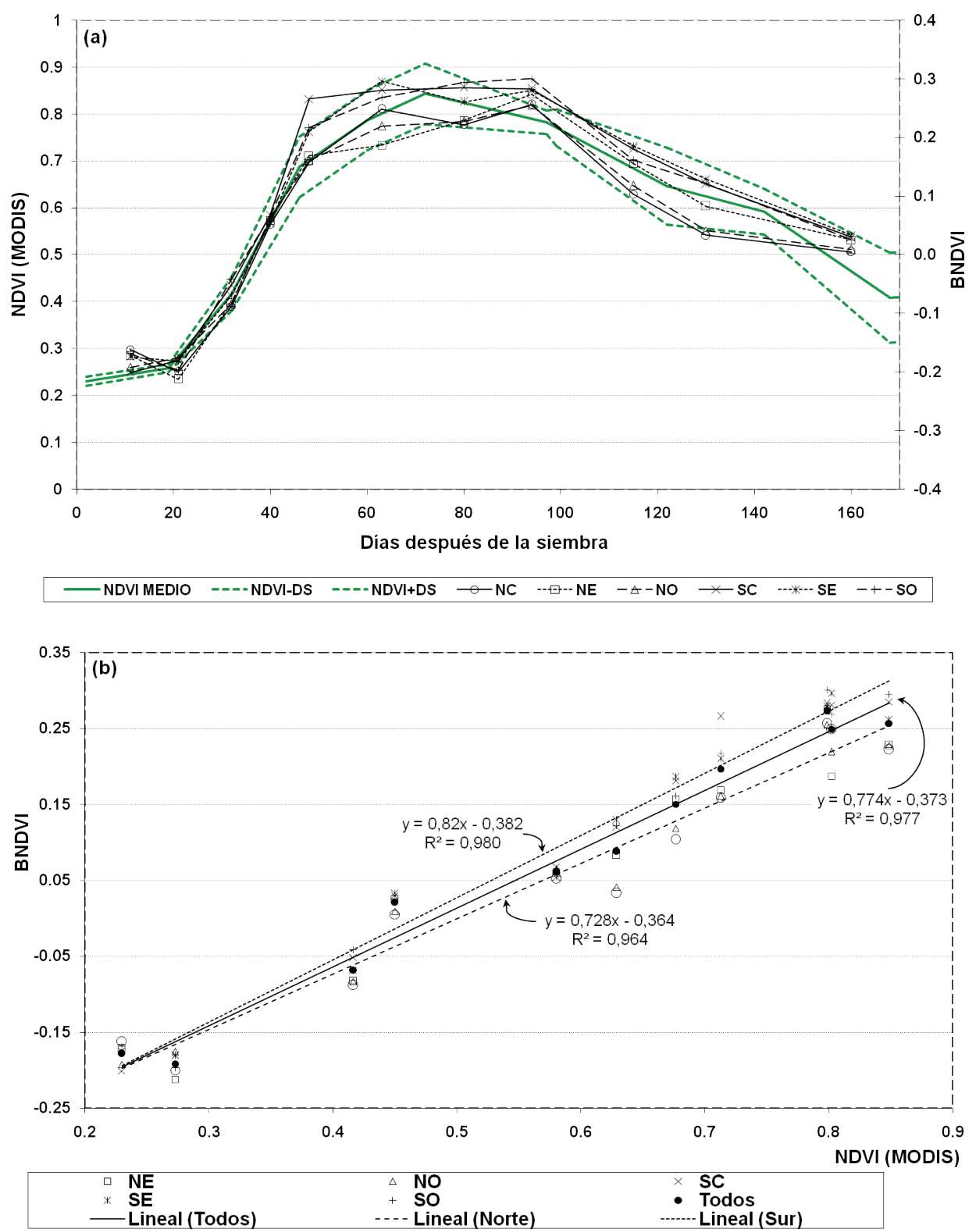

Figura 5. a) Variación del NDVI respecto a los días después de la siembra obtenidos alternativamente por el sistema de teledetección AQUA-MODIS (valor medio +/- el desvío estándar (DS)) y de BNDVI obtenido por la cámara digital modificada en cada uno de los sectores monitoreados del lote. b) Relación lineal entre los valores de NDVI de AQUA-MODIS y BNDVI para los sectores agrupados.

sobre el cultivo. Debido a la diferente escala numérica de cada dispositivo, cada uno se presenta en un eje de ordenada distinto.

Si bien existe un marcado contraste de resolución entre ambas fuentes de datos, es factible ajustar la escala gráfica del BNDVI obtenido a campo de modo que los valores del conjunto de sectores de muestreo quedan circunscriptos estrictamente por la banda +/- un desvío estándar alrededor del NDVI medio de MODIS, que es representativo del comportamiento general del lote. La relación entre el NDVI de MODIS y BNDVI obtenido a campo muestra un comportamiento lineal en cada uno de los sectores y una correlación altamente significativa $(P<0,01)$ en todos los casos. De esta forma, se verifica que ambas fuentes de datos 
producen resultados similares a los efectos de establecer diferencias de CC y fueron sensibles para evaluar el comportamiento desigual ocasionado por el manejo sitio-específico del lote.

La Figura 5 (b) presenta la relación establecida al agrupar la información de los tres sectores del sur y los tres del norte. De esta forma, se verifica que durante la etapa vegetativa existen diferencias menos notables en el desarrollo foliar entre los distintos sectores y, asimismo, que la variabilidad espacial en el lote y el contraste entre los sectores de muestreo son más notables durante la etapa reproductiva. Es destacable que, mientras la función lineal establecida entre los datos de BNDVI local y el NDVI medio de MODIS de los sectores ubicados al norte del lote se presenta por debajo del comportamiento medio, la correspondiente al sector sur está por encima, conforme al diferente manejo aplicado en el lote (Figura 5a). Sakamoto et al. (2012) también obtuvieron relaciones muy consistentes entre indicadores espectrales de la vegetación obtenidos con cámaras convencionales y los producidos rutinariamente por MODIS. En este sentido, Li et al. (2010) determinaron que la información de CC obtenida de cámaras digitales comerciales resultó igual de robusta que la obtenida a partir de instrumentos más específicos (GreenSeeker ${ }^{\circledR}$ ) a los efectos de evaluar el contenido de nitrógeno de los cultivos.

Más allá del uso de los datos de NDVI para estimar en este caso CC del maíz, a los efectos de producir información más específica y de mayor alcance agronómico, la aptitud puesta de manifiesto por la cámara modificada avala el empleo de estos registros espectrales para identificar diferencias productivas dentro de un lote, y ratifica el empleo de la teledetección como un complemento fundamental de la AP (Chang et al., 2003; Hunt et al., 2005; Hunt et al., 2010).

$\mathrm{Si}$ se considera a la radiación solar fotosintéticamente activa (RFA) que se acumula durante el ciclo un indicador de la capacidad potencial de producción de biomasa (Sinclair y Muchow, 1999), y se asume que CC representa directamente la fracción de radiación interceptada por el cultivo (Steven et al., 1986; Purcell, 2000), las relaciones lineales de la Figura 6 (a) describen el comportamiento particular de cada sector, resultando las pendientes de los sectores hacia el norte notablemente menores a las del sur. La pendiente de esta relación es una aproximación al valor de eficiencia en el uso de la radiación (EUR) que, en los sectores SO y SE, alcanza una magnitud de $3 \mathrm{~g} \mathrm{MJ}^{-1}$. Con un rango de EUR comprendido entre 3 y $4 \mathrm{~g} \mathrm{MJ}^{-1}$ para cultivos de maíz bajo condiciones potenciales (Sinclair y Muchow, 1999;
Lindquist et al., 2005), el valor de eficiencia que arroja esta experiencia se encuentra próximo al límite inferior. Por su parte, las tasas de eficiencia para los sectores al norte del lote sólo alcanzan valores entre 2,3 y 2,5 $\mathrm{g} \mathrm{MJ}^{-1}$, lo que denota una restricción sobre la producción de biomasa.

Por su parte, la Figura 6 (b) presenta la relación entre los rendimientos de grano observados y estimados en cada sector a partir de las tasas de conversión y los valores de radiación solar fotosintéticamente activa interceptada (RFAI), considerando un índice de cosecha de 0,48 de acuerdo a Sinclair et al. (1990). Además, se presenta la relación con el valor de rendimiento potencial, que se estimó utilizando un valor de EUR de 3,8 $\mathrm{g} \mathrm{MJ}^{-1}$ (Lindquist et al., 2005). Ambas figuras ponen de manifiesto que la productividad de biomasa y rendimiento de grano se presentan por debajo de las tasas potenciales, indicando la ocurrencia de contingencias desfavorables durante el ciclo de cultivo, además de las diferencias de CC producidas por la densidad de siembra inicial.

A los efectos de explicar en primer lugar las variaciones de CC durante el ciclo, y de su repercusión sobre la productividad luego, la Figura 7 muestra la evolución temporal en el contenido de agua que se registraron entre los distintos sectores en el transcurso del monitoreo. Tanto al comienzo del ciclo (los primeros tres muestreos) como hacia su finalización los sectores presentaron contenidos de agua similares. Las Iluvias incesantes al final del ciclo incrementaron el contenido de agua hasta valores próximos a capacidad de campo y homogeneizaron su disponibilidad en todo el lote (Figura 2). El contenido medio de agua del perfil respecto al de capacidad de campo fue notoria y significativamente más bajo $(P<0,01)$ en los tres sectores del norte a los 80 y 115 días después de la siembra, es decir en plena etapa reproductiva del maíz, coincidiendo con el período en que disminuyeron las precipitaciones y se debió aplicar riego suplementario. El contenido de agua del perfil en los sectores hacia el norte se redujo por debajo de $60 \%$ de capacidad de campo en R1 y resultó menor a 40\% en R4, lo que explica en parte la merma productiva (Meyer et al., 1993). Asimismo, la menor disponibilidad de agua se expresa reduciendo los valores de CC, que se amplifica a partir de 80 días después de la siembra (Figura 4).

La menor producción del cultivo de maíz en los sectores ubicados al norte del lote se explica entonces por un menor valor de CC que, asociado inicialmente a la densidad de siembra más baja (Figura 2), se amplifica luego por efecto de la menor disponibilidad de agua en R1 y R4. Además de la 

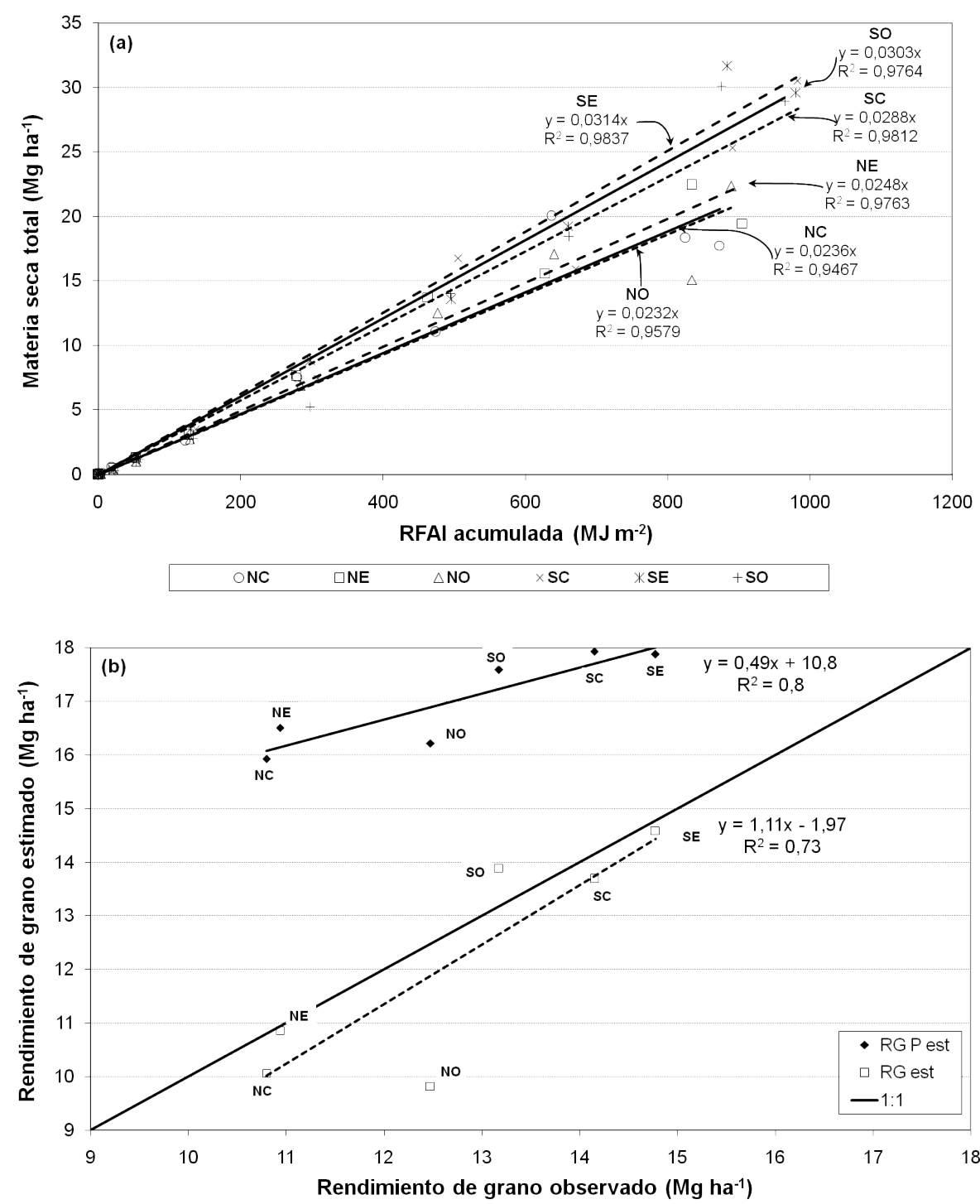

Figura 6. (a) Relación entre la radiación fotosintéticamente activa interceptada (RFAl) y la materia seca acumulada durante el ciclo de maíz 2013-2014 en los sectores monitoreados del lote. (b) Relación entre los valores de rendimiento de grano observado y estimados (RG est) y los de carácter potencial (RG P) en cada sector.

caída anticipada de CC que se registró al norte del lote, de acuerdo al momento de ocurrencia de la falta de agua es probable que otros componentes del rendimiento (número de granos o su peso) también se hayan visto afectados por el estrés hídrico (Andrade et al., 1999; Earl \& Davis, 2003).

Finalmente, la Figura 8 presenta el mapa de rendimiento de maíz del lote, que se depuró de valores extremos y fue suavizado con un filtro de mediana, con los valores de productividad observados superpuestos en cada uno de los sectores de muestreo. Si bien el mapa pone de manifiesto una condición de variabilidad productiva importante, el rendimiento de los sectores localizados al norte presentan un valor medio significativamente inferior $(P<0,001)$ a los del sur. Si se considera que el ingreso de agua al lote fue uniforme, tanto por el aporte natural de la lluvia como la provista por el riego, un factor determinante de las diferencias de humedad del suelo entre sectores es factible que ocurra principalmente como consecuencia de la redistribución particular 


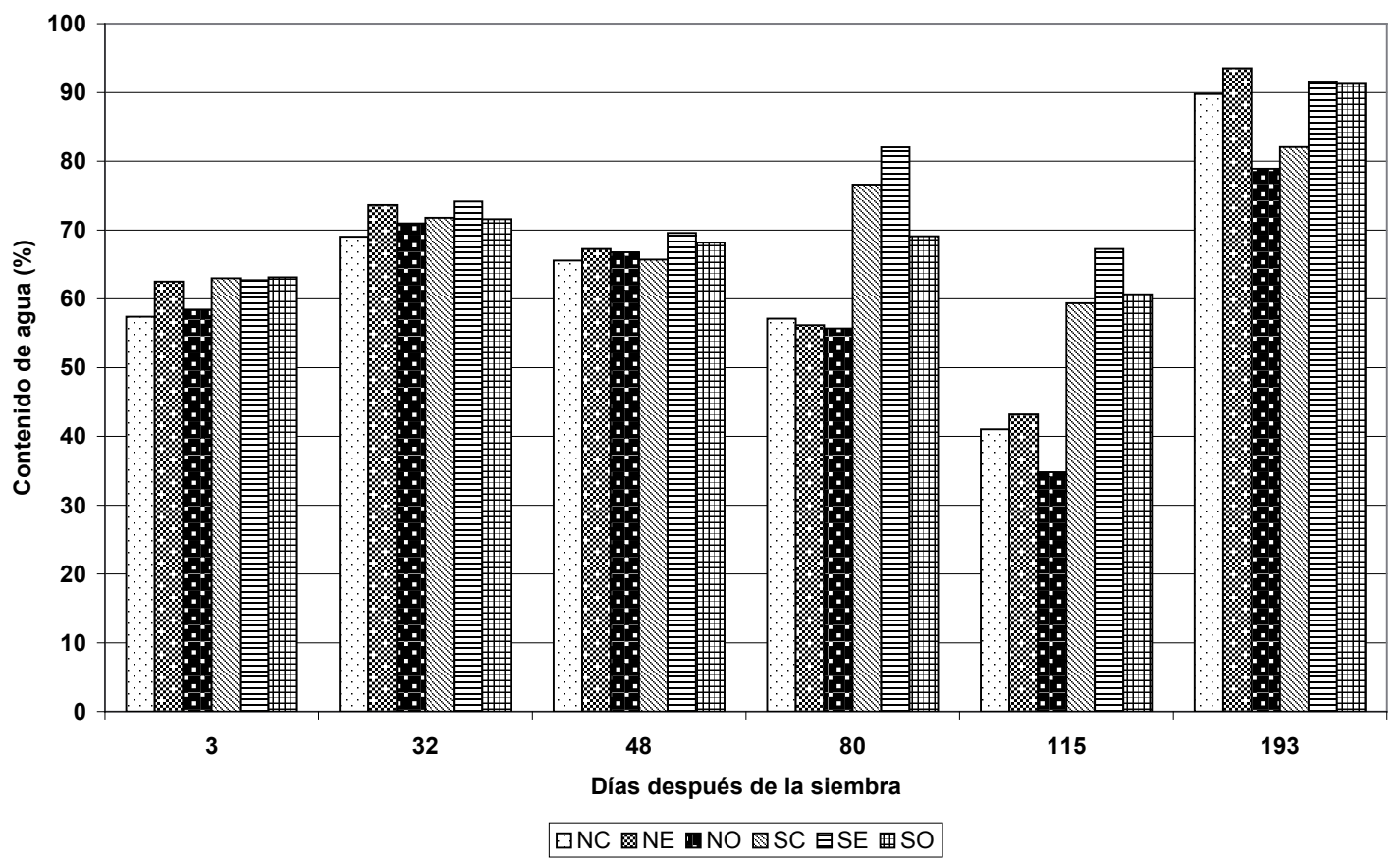

Figura 7. Contenido de agua del suelo en el perfil (0-1,20 m) con relación al valor a capacidad de campo durante el ciclo en los sectores monitoreados del lote de maíz.

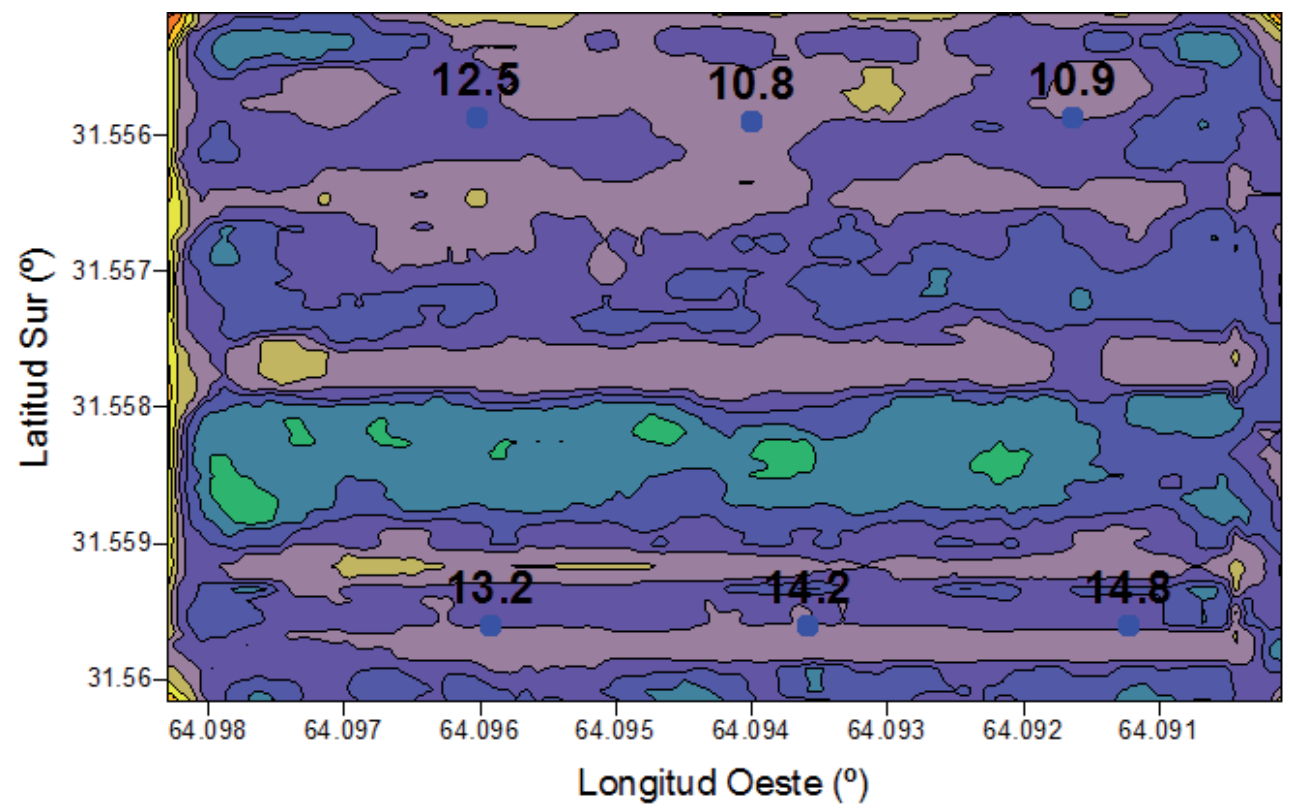

Rendimiento $\left(\mathrm{Mg} \mathrm{ha}^{-1}\right.$ )

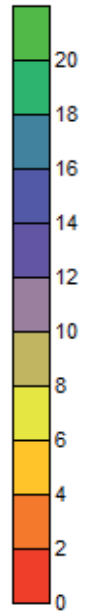

Figura 8. Mapa de rendimientos de grano del lote de maíz en 2013-2014 y los valores observados en cada uno de los sectores monitoreados. 
del agua asociada a pequeñas diferencias topográficas y de microescala en el lote. Por otra parte, la configuración del mapa muestra que las variaciones de rendimiento siguen en buena medida el ordenamientro de los surcos de riego, lo que denota diferencias operativas respecto al manejo del agua. En este sentido, es indudable que una parte importante de la variabilidad en los mapas de rendimiento es producida principalmente por la variación de las propiedades que afectan la disponibilidad de agua (Timlin et al., 2001; Kitchen et al., 2003).

\section{CONCLUSIONES}

La CC de maíz resultó un parámetro apropiado para evaluar diferencias de la capacidad de producción de biomasa y rendimiento en un lote de maíz sembrado a tasa variable. El umbral utilizado para clasificar como vegetación o suelo cada píxel de la imagen de NDVI que, durante la etapa vegetativa asume un valor de 0,0, se aumentó a 0,1 en la etapa reproductiva para representar de manera más fidedigna el tejido verde en la escena hacia el final del ciclo. Los datos de NDVI de alcance local obtenidos con una cámara digital modificada resultaron muy consistentes con respecto a los valores que produce el sistema de teledetección AQUA-MODIS, información que permitió reconocer un potencial productivo diferente entre los sectores norte y sur monitoreados en el lote. Este comportamiento productivo se corroboró a partir de menores valores observados de biomasa y rendimiento de grano de los sectores ubicados al norte, debido a la menor CC y una EUR más reducida. A partir de una menor densidad se siembra, la disminución de producción que acusan los sectores al norte del lote se explica asimismo en razón del contenido de agua del suelo más bajo durante R1 y R4 que, no sólo redujo la intercepción de radiación y la EUR, también comprometió a otros componentes del rendimiento de maíz. El contraste de la disponibilidad de humedad del suelo en el lote puede estar asociado tanto a diferencias topográficas y de microrelieve que modifican la redistribución de agua en el terreno, como a la operación del riego, aspecto que se debe dilucidar con mayor detalle.

\section{AGRADECIMIENTO}

Los autores agradecen a la Secretaría de Ciencia y Técnica de la Universidad Nacional de Córdoba por el apoyo económico recibido.

\section{BIBLIOGRAFÍA}

Andrade, F.H., C. Vega, S.A. Uhart, A. Cirilo, M. Cantarero, and O. Valentinuz, 1999. Kernel number determination in maize. Crop Sci. 39:453-459.

Chang, J., D.E. Clay, K. Dalsted, S. Clay, and M. O'Neill, 2003. Corn (Zea mays L.) yield prediction using multispectral and multidate reflectance. Agron. J. 95:1447-1453.

Clay, D.E., K.-I. Kim, J. Chang, S.A. Clay, and K. Dalsted, 2006. Characterizing water and nitrogen stress in corn using remote sensing. Agron. J. 98:579-587.

de la Casa, A., G. Ovando, L. Bressanini, Á. Rodríguez, y J. Martínez, 2007. Uso del índice de área foliar y del porcentaje de cobertura del suelo para estimar la radiación interceptada en papa. Agricultura Técnica 67(1):78-85.

de la Casa, A., G. Ovando, L. Bressanini, J. Martínez y Á. Rodríguez, 2011. Eficiencia en el uso de la radiación en papa estimada a partir de la cobertura del follaje. AGRISCIENTIA 28(1):21-30.

de la Casa, A., G. Ovando, L. Bressanini, y J. Martínez, 2013b. Estimación de la cobertura del suelo y evaluación de la fertilización en papa con registros de cámaras digitales convencionales y modificadas. Anales 42 Jornadas Argentinas de Informática 94104. ISSN 1852-4850.

de la Casa, A.C., G.G. Ovando, A.C. Ravelo, E.G. Abril, and $\mathrm{H}$. Bergamaschi, 2013a. Estimating maize ground cover using spectral data from Aqua-Modis in Córdoba, Argentina. International Journal of Remote Sensing 35(4):1295-1308.

Doraiswamy, P.C., J.L. Hatfield, T.J. Jackson, J.H. Prueger, B. Akhmedov, and A.J. Stern, 2004. Crop condition and yield simulations using Landsat and MODIS imagery. Rem. Sen. Environ. 92:548-559.

Earl, H.J., and R.F. Davis, 2003. Effect of drought stress on leaf and whole canopy radiation use efficiency and yield of maize. Agron. J. 95:688-696.

EOS, 2014. NASA Earth Observing System data gateway. Disponible en:

<https://lpdaac.usgs.gov/dataset_discovery/modis/ modis_products_table/myd13q1>

Consultada el 20/02/2017

Gitelson, A.A., Y.J. Kaufman, R. Stark, and D. Rundquist, 2002. Novel algorithms for remote estimation of vegetation fraction. Remote Sens. Environ. 80:76-87.

Gitelson, A.A., 2004. Wide Dynamic Range Vegetation Index for remote quantification of biophysical characteristics of vegetation. J. Plant Physiol. 161:165173.

Hatfield, J.L., A.A. Gitelson, J.S. Schepers, and C.L. Walthall, 2008. Application of spectral remote sensing for agronomic decisions. Agron. J. 100:S117-S131.

Huete A., K. Didan, T. Miura, E.P. Rodríguez, X. Gao, and L.G. Ferreira, 2002. Overview of the radiometric and 
biophysical performance of the MODIS vegetation indices. Rem. Sen. Environ. 83:195-213.

Hunt, E.R.Jr., M. Cavigelli, C.S.T. Daughtry, J. McMurtrey III, and C.L. Walthall, 2005. Evaluation of digital photography from model aircraft for remote sensing of crop biomass and nitrogen status. Precision Agriculture 6:359-378.

Hunt, E.R.Jr., W.D7. Hively, S.J. Fujikawa, D.S. Linden, C.S.T. Daughtry, and G.W. McCarty, 2010. Acquisition of NIR-Green-Blue digital photographs from unmanned aircraft for crop monitoring. Remote Sens. 2:290-305. doi:10.3390/rs2010290.

Inoue, Y., 2003. Synergy of remote sensing and modeling for estimating ecophysiological processes in plant production. Plant Prod. Sci. 6:3-16.'

Kool, D., N. Agam, N. Lazarovitch, J.L. Heitman, T.J. Sauer, and A. Ben-Gal, 2014. A review of approaches for evapotranspiration partitioning. Agricultural and Forest Meteorology 184:56-70.

Li, Y., D. Chen, C.N. Walker, and J.F. Angus, 2010. Estimating the nitrogen status of crops using a digital camera. Field Crops Research 118:221-227.

Lindquist, J.L., T.J. Arkebauer, D.T. Walters, K.G. Cassman, and A. Dobermann, 2005. Maize radiation use efficiency under optimal growth conditions. Agron. J. 97:72-78.

Maas, S.J., 1993. Within-season calibration of modeled wheat growth using remote sensing and field sampling. Agron. J. 85:669-672.

Maas, S.J., 1998. Estimating cotton canopy ground cover from remotely sensed scene reflectance. Agron. J. 90:384-388.

Maas, S.J., 2000. Linear mixture modeling approach for estimating cotton canopy ground cover using satellite multispectral imagery. Remote Sensing of Environment 72:304-308.

Maas, S.J. and N. Rajan, 2008. Estimating ground cover of field crops using medium-resolution multispectral satellite imagery. Agron. J. 100:320-327.

Martin, K.L., K. Girma, K.W. Freeman, R.K. Teal, B. Tuba a, D.B. Arnall, B. Chung, O. Walsh, J. B. Solie, M. L. Stone, and W. R. Raun, 2007. Expression of variability in corn as influenced by growth stage using optical sensor measurements. Agron. J. 99:384-389.

Meyer, S.J., K.G. Hubbard, and D.A. Wilhite, 1993. A cropspecific drought index for corn: I. Model development and validation. Agron. J. 86:388-395.

Moran, M.S., Y. Inoue, and E.M. Barnes, 1997. Opportunities and limitations for image-based remote sensing in precision crop management. Remote Sens. Environ. 61:319-346.

Mulla, D.J. and J.S. Schepers, 1997. Key processes and properties for site-specific management. In "The State of Site-Specific Management for Agriculture" (F. J. Pierce and E. J. Sadler, Eds.), ASA Miscellaneous Publication, pp. 1-18. ASA, CSSA, and SSSA,
Madison, WI.

Kitchen, N.R., S.T. Drummond, E.D. Lund, K.A. Sudduth, and G.W. Buchleiter, 2003. Soil electrical conductivity and topography to yield for three contrasting soil-crop systems. Agron. J. 95:483-495

Pierce, F.J. and P. Nowak, 1999. Aspects of precision agriculture. Advanced in Agronomy 67:1-85.

Purcell, L.C., 2000. Soybean canopy coverage and light interception measurements using digital imagery. Crop Science 40:834-837

Raes, D., P. Steduto, T.C. Hsiao, and E. Fereres, 2009. AquaCrop-The FAO crop model to predict yield response to water: II Main algorithms and software description. Agron. J. 101:438-44.

Ritchie, S.W., J.J. Hanway, and G.O. Benson. 1992. How a corn plant develops. Special Report N48. http:// maize.agron.iastate.edu/corngrows.html. Iowa State University, Cooperative Extension Service, Ames, IA.

Rollán, A.A. y O.A. Bachmeier, 2014. Compactación y retención hídrica en Haplustoles de la provincia de Córdoba (Argentina) bajo siembra directa. AGRISCIENTIA 31(1):1-10.

Sakamoto, T., A.A. Gitelson, A.L. Nguy-Robertson, T.J. Arkebauer, B.D. Wardlow, A.E. Suyker, S.B. Verma, and M. Shibayama, 2012. An alternative method using digital cameras for continuous monitoring of crop status. Agricultural and Forest Meteorology 154155:113-126

Sibley, A.M., P. Grassini, N.E. Thomas, K.G. Cassman, and D.B. Lobell, 2013. Testing remote sensing approaches for assessing yield variability among maize fields. Agron. J. 106:24-32.

Sinclair, T.R., J.M. Bennett, and R.C. Muchow, 1990. Relative sensitivity of grain yield and biomass accumulation to drought in field-grown maize. Crop Sci. 30:690-693.

Sinclair, T.R., and R.C. Muchow, 1999. Radiation use efficiency. Adv. Agron. 65:216-265.

Steven, M.D., P.V. Biscoe, K.W. Jaggard, and J. Paruntu, 1986. Foliage cover and radiation interception. Field Crops Research 13:75-87.

Teal, R.K., B. Tubana, K. Girma, K.W. Freeman, D.B. Arnall, O. Walsh, and W.R. Raun, 2006. In-season prediction of corn grain yield potential using normalized difference vegetation index. Agron. J.98:1488-1494.

Timlin, D., Y. Pachepsky, C. Walthall, and S. Loechel, 2001. The use of a water budget model and yield maps to characterize water availability in a landscape. Soil \& Tillage Research 58:219-231.

Viña, A., A.A. Gitelson, D.C. Rundquist, G. Keydan, B. Leavitt, and J. Schepers, 2004. Monitoring maize (Zea mays L.) phenology with remote sensing. Agron. J. 96:1139-1147.

Von Bueren, S.K.; A. Burkart, A. Hueni, U. Rascher, M.P. Tuohy and I.J. Yule, 2015. Deploying four optical UAV-based sensors over grassland: challenges and 
limitations. Biogeosciences 12(1):163-175.

Wang, F.M.; J.F. Huang, Y.L. Tang and X.Z. Wang, 2007. New vegetation index and its application in estimating leaf area index of rice. Rice Science 14(3):195-203.

Woebbecke, D. M.; Meyer, G. E., Von Bargen, K., and D.A. Mortensen, 1995. Color indices for weed identification under various soil, residue, and lighting conditions. Transactions of the ASAE-American Society of Agricultural Engineers, 38(1): 259-270.

Woli, K.P., C.L. Burras, L.J. Abendroth, and R.W. Elmore,
2014. Optimizing corn seeding rates using a field's corn suitability rating. Agron. J. 106:1523-1532.

Xiong X. and Barnes W., 2003. Early On-orbit Calibration Results from Aqua MODIS Proceedings of SPIE Volume 4881 Sensors, Systems, and Next-Generation Satellites VI, Hiroyuki Fujisada, Joan B. Lurie, Michelle L. Aten, Konradin Weber, Editors, April 2003, Vol. 4881 pp. 327-336 Sep. 23-26: SPIE Europe - Agia Pelagia, Crete. 